\title{
Frequency and clinical features of BRAF mutations among patients with stage III/IV lung adenocarcinoma without EGFR/ALK aberrations
}

This article was published in the following Dove Press journal: OncoTargets and Therapy

\author{
Ho Cheol Kim' \\ Yeh Rim Kang ${ }^{2}$ \\ Wonjun Ji' \\ Yeon Joo Kim' \\ Shinkyo Yoon ${ }^{3}$ \\ Jae Cheol Lee ${ }^{3}$ \\ Chang-Min Choi ${ }^{1,3}$ \\ 'Department of Pulmonary and Critical \\ Care Medicine, Asan Medical Center, \\ University of Ulsan College of Medicine, \\ Seoul, South Korea; ${ }^{2}$ Medical \\ Department, Oncology, Novartis Korea \\ Pharmaceuticals, Seoul, South Korea; \\ ${ }^{3}$ Department of Oncology, Asan Medical \\ Center, University of Ulsan College of \\ Medicine, Seoul, South Korea
}

Purpose: BRAF mutations are found in $1-5 \%$ of non-small cell lung cancers, particularly adenocarcinomas. However, information regarding this mutation is limited in patients without EGFR/ALK aberrations, who have limited treatment options.

Patients and methods: The medical records of 224 stage III/IV adenocarcinoma patients without EGFR/ALK aberrations and with available pathologic tissue, were retrospectively reviewed. BRAF mutations were evaluated using a PNAClamp ${ }^{\mathrm{TM}}$ BRAF mutation detection kit (Panagene, Daejeon, Korea). The outcomes in the study population were compared with stage III/IV adenocarcinoma patients harboring an EGFR mutation. A case report of targeted therapy against BRAF mutations was also presented.

Results: A cohort of 222 adenocarcinoma patients with adequate pathologic tissue samples was analyzed. The median patient age was 63 years, $68.8 \%$ of the patients were male and $68.7 \%$ were ever-smokers. The V600E BRAF mutation was detected in 4 patients $(1.8 \%)$. The 222 study patients had a poorer survival outcome compared to stage III/IV adenocarcinoma patients with an EGFR mutation (median, 12 vs 67 months, $P<0.001$ ) from a recent previous study. Moreover, a 47-year-old female with a recurrent adenocarcinoma and a BRAF V600E mutation exhibited tumor regression after a fourth line therapy with dabrafenib and trametinib, targeting agents against BRAF mutations.

Conclusion: Although BRAF mutations are found in $1.8 \%$ of advanced adenocarcinoma patients without EGFR/ALK aberration, they may be able to serve as a treatment target in those patients.

Keywords: BRAF, adenocarcinoma, lung cancer

\section{Introduction}

Lung cancer is a leading cause of cancer-related mortality, causing approximately $25 \%$ of cancer deaths. ${ }^{1}$ Specific genetic aberrations and diverse molecular pathways have been recently described in lung tumors, which has led to the development of numerous targeted therapies. ${ }^{2}$ As a representative example, epidermal growth factor receptor (EGFR)-targeted tyrosine kinase inhibitors are an important established treatment option for patients with EGFR mutation-positive tumors. ${ }^{3,4}$ In addition, anaplastic lymphoma kinase (ALK) inhibitors have been found to be effective in non-small cell lung cancer (NSCLC) patients with ALK rearrangements. ${ }^{5}$

In addition to EGFR mutations and ALK rearrangements, genomic analyses have identified other driver mutations in the pathogenesis of lung cancer. ${ }^{6}$ One is the B-Raf and V-Raf murine sarcoma viral oncogene homolog (BRAF), which
Correspondence: Chang-Min Choi Department of Pulmonary and Critical Care Medicine, Asan Medical Center, University of Ulsan College of Medicine, 88 Olympic-ro 43-gil, Songpa-gu, Seoul 05505 , South Korea

Tel +82 230105902

Fax +82 230105902

Email ccm@amc.seoul.kr 
encodes a serine-threonine kinase within the MAPK signaling pathway. ${ }^{7}$ BRAF mutations were initially detected in approximately $50 \%$ of melanomas ${ }^{8}$ and are regarded as important therapeutic targets for patients diagnosed with that type of cancer. ${ }^{9,10}$ BRAF mutations have also been detected in 1 to $5 \%$ of NSCLCs, particularly in adenocarcinomas. $^{11,12}$ Although there have been several previous reports on BRAF mutations in Asian lung cancer cohorts, ${ }^{13-15}$ data on these mutations are lacking in adenocarcinoma patients without EGFR/ALK aberrations, who are known to have limited treatment options. Our present study aims to investigate the incidence of BRAF mutations among advanced adenocarcinoma patients without EGFR/ALK aberrations and analyze the clinical characteristics of these cases.

\section{Materials and methods}

\section{Study populations and methods}

This retrospective cohort study was performed at the Asan Medical Center, a tertiary referral hospital in Seoul, South Korea. The study population was comprised of 224 patients with stage III/IV lung adenocarcinoma diagnosed between July 2010 and June 2017, which did not have EGFR/ALK aberrations and for which pathologic tissue samples were available. Two patients in the initial cohort of 224 cases had insufficient pathologic tissue and were excluded from further analysis. The included patients were classified into two groups based on their BRAF mutation status. The study flow chart is shown in Figure 1. Clinical data regarding patient age, sex, smoking history, clinical stage (according to the seventh edition of the TNM International Staging System), treatment modality, results of molecular tests for BRAF mutation and survival data for the included patients were retrospectively collected from medical records, telephone interviews, and/or records from the National Health Insurance of Korea. The study protocol was approved by the Institutional Review Board of Asan Medical Center (approval number: 2018-1109) and written informed consent for the use of tissue samples for clinical research was obtained from all patients or their appropriate family members. The prognoses of the study patients were compared with those of patients with stage III/IV lung adenocarcinoma which harbored an EGFR mutation, which had been described in our previous study. ${ }^{16}$

\section{Evaluation of BRAF mutations}

We used the PNAClamp ${ }^{\mathrm{TM}}$ BRAF mutation detection kit (Panagene, Daejeon, Korea) to detect the BRAF V600E mutation. This kit is based on peptide nucleic acid-mediated real-time PCR clamping technology. All reactions were conducted in a final volume of $20 \mu \mathrm{L}$ and contained template DNA, primers, PNA probe, and a SYBR green PCR master mix. Real-time PNA-clamping PCR was performed using a CFX96 real-time PCR system (Bio-Rad, Pleasanton, CA). The cycling conditions included a 5 min hold at $94{ }^{\circ} \mathrm{C}$ followed by 40 cycles of 30 seconds at $94{ }^{\circ} \mathrm{C}, 20$ seconds at $70{ }^{\circ} \mathrm{C}, 30$ seconds at $63{ }^{\circ} \mathrm{C}$, and 30 seconds at $72{ }^{\circ} \mathrm{C}$. In the assay, the PNA probe and primers were represented by separate oligonucleotides, with the PNA probe recognizing a region located between the regions of the template complementary to the forward and reverse primers. Positive signals were detected via the intercalation of the SYBR green fluorescent dye. The PNA probe sequences, which were complementary to the wild-type BRAF (V600E), enhanced the preferential amplification of mutant sequences by competitively inhibiting the amplification of wild-type sequences (Figure 2). PCR efficiency was determined by measuring the cycle threshold (CT) value. CT values for the control and mutation assays were obtained by observing the

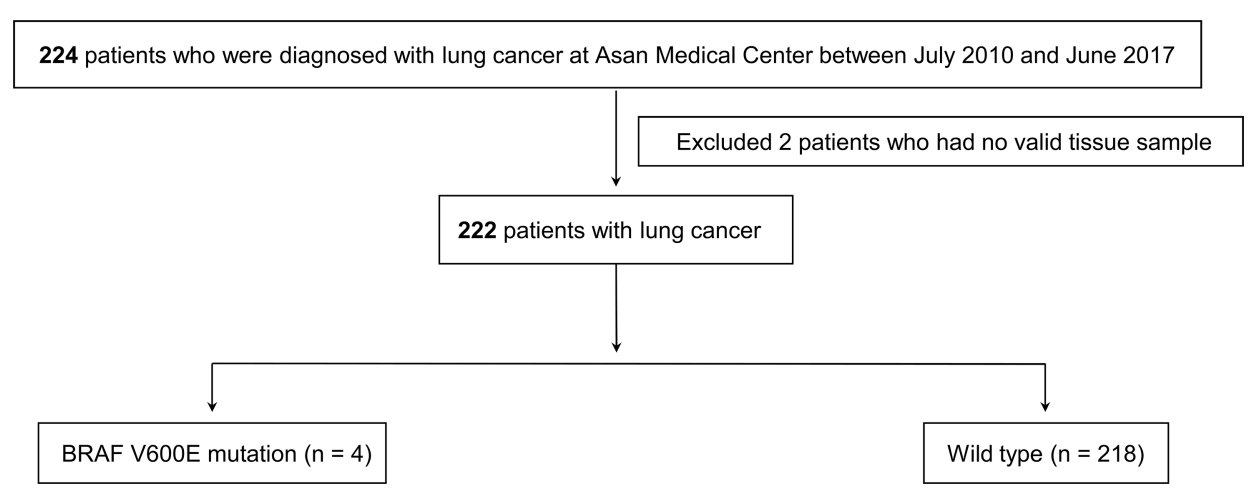

Figure I Study flow chart. 


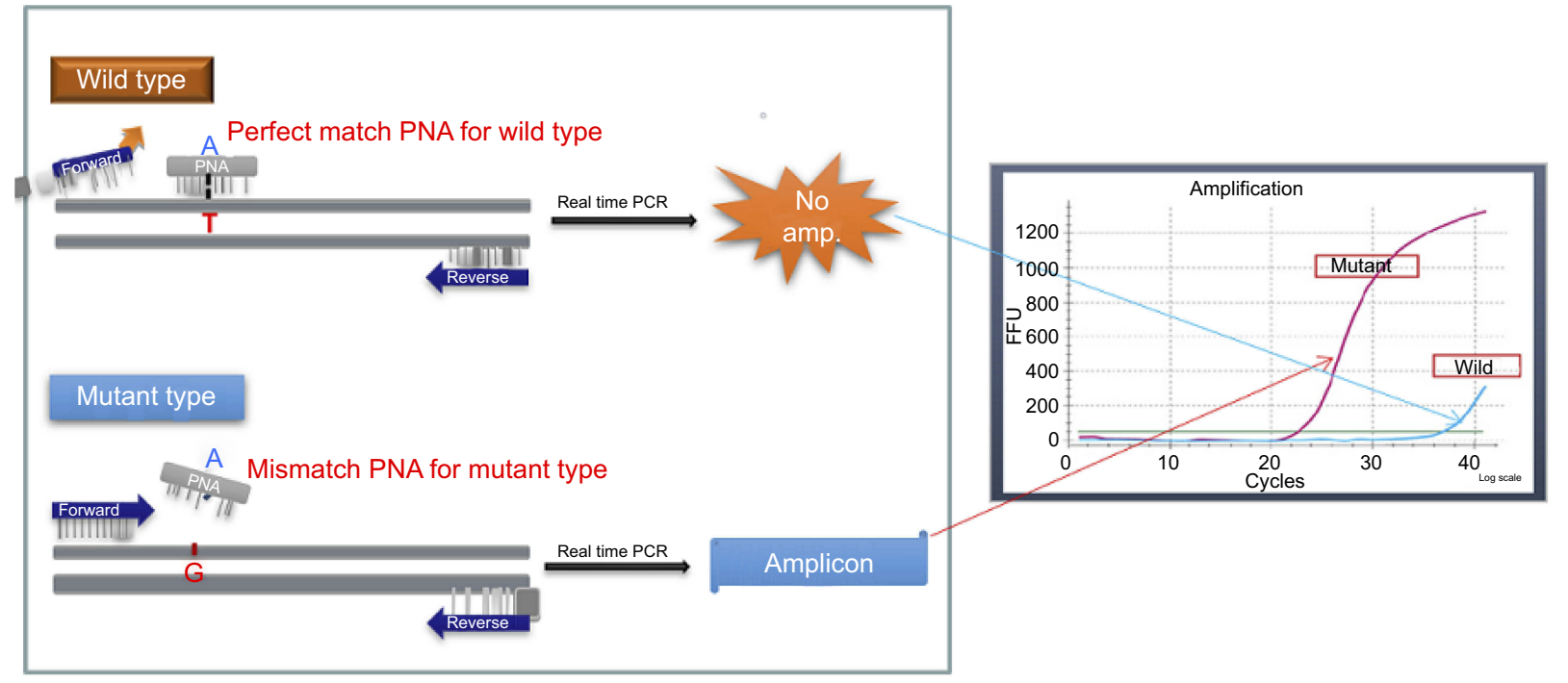

Figure 2 Principle of the PNAClamp ${ }^{\text {TM }}$ BRAF mutation detection kit.

SYBR green amplification plots. The delta CT $(\Delta \mathrm{CT})$ value was calculated by subtracting the $\mathrm{CT}$ value of a tested sample from the standard $\mathrm{CT}$ value of a clamping control sample $([$ Standard CT $]-[$ Sample CT $]=\Delta \mathrm{CT}-1)$. The sample was considered positive if the value of $\Delta \mathrm{CT}-1$ was greater than 2 , as verified in a previous study. ${ }^{17}$

\section{Statistical analysis}

Continuous variables were expressed as the mean \pm standard deviation (SD) or median (interquartile range [IQR]), and categorical variables as percentages. Continuous variables were compared using a Mann-Whitney U test, and categorical variables using either a chi-square or Fisher's exact test, as appropriate. Risk factors for mortality were analyzed using Cox proportional hazards models. Variables with a $P$-value $<0.10$ on univariate analysis were used in the multivariate analysis. Survival was analyzed by the KaplanMeier method and compared by log-rank tests. All $P$-values were two-tailed, with a $P$-value of $<0.05$ being considered statistically significant. All statistical analyses were performed using SPSS 20.0 (IBM Corporation, Armonk, NY).

\section{Results}

\section{Patient characteristics}

A total of 222 patients with lung cancer, without EGFR/ ALK aberrations, and with adequate pathologic sample tissues were included in this study. The baseline and clinical characteristics of these patients are summarized in Table 1. The median patient age was 63 years (interquartile range, $57-71$ years), with $68.8 \%$ of patients being

Table I Baseline and clinical characteristics of the study patients in accordance with their BRAF V600E mutation status

\begin{tabular}{|c|c|c|c|c|}
\hline & Total & BRAF V600E mutation & BRAF wild type & $P$-value \\
\hline Patients number & 222 & 4 & 218 & \\
\hline Age, years & $63[57-7 I]$ & $71[58-76]$ & $63[57-7 I]$ & 0.309 \\
\hline Male sex & I54 (68.8) & $3(75.0)$ & $150(68.8)$ & $>0.999$ \\
\hline Ever-smoker $(n=20 I)$ & $138(68.7)$ & $2(50.0)$ & $136(69.0)$ & 0.591 \\
\hline Clinical stage & & & & $>0.999$ \\
\hline III & $54(24.3)$ & I (25.0) & $53(24.3)$ & \\
\hline IV & $168(75.7)$ & $3(75.0)$ & $165(75.7)$ & \\
\hline Surgery & $24(10.8)$ & I (25.0) & $23(10.6)$ & 0.369 \\
\hline Chemotherapy & $156(70.3)$ & $3(75.0)$ & $153(70.2)$ & $>0.999$ \\
\hline Radiation therapy & $92(41.4)$ & 0 & $92(42.2)$ & 0.144 \\
\hline Best supportive care & $38(17.4)$ & 0 & $38(17.1)$ & $>0.999$ \\
\hline
\end{tabular}

Note: Data are presented as a median (interquartile range), or number (\%), unless otherwise indicated. 
male, $=68.7 \%$ being ever-smokers. Patients included in the study had either stage III (24.3\%) or stage IV (75.7\%) lung cancer. The treatments received at initial diagnosis by the patients in the study cohort included chemotherapy (70.3\%), radiation therapy (41.4) or surgery $(10.8 \%)$. By using PNA-clamping PCR, we detected a BRAF mutation in 4 patients (1.8\%), all of whom had the V600E mutation (Figure 3). Notably, there were no differences in patient characteristics between subjects with and without this BRAF mutation.

\section{Risk factors for mortality in the study population}

One hundred and seventy-nine (80.6\%) patients died during follow-up. By using univariate Cox analysis, we determined that male sex (hazard ratio [HR], 1.503; 95\% confidence interval $[\mathrm{CI}], 1.083-2.087, P=0.015)$ and best supportive care (HR, 1.639; 95\% CI, 1.111-2.416, $P=0.013$ ) were significant predictors of mortality (Table 2 ). In addition, old age and ever-smoker status were marginally associated with a poorer prognosis. However, after performing multivariate analysis, our results showed that only best supportive care (HR, 2.252; 95\% CI, 1.408-3.600, $P=0.001$ ) was an independent predictor of mortality (Table 2 ).

\section{Comparison between the study population and patients with an EGFR mutation}

We compared the baseline characteristics of our current study cohort of 222 patients with our previously reported cohort of 239 stage III/IV adenocarcinoma patients with an EGFR mutation (Table 3). The patients included in this study were older, more likely to be male and ever-smokers, and had a less advanced disease stage when compared with the previously analyzed cohort. Kaplan-Meier survival analysis further revealed that the patients included in this study had a significantly poorer survival outcome that the previously analyzed cohort. (median survival: 12 vs 67 months, $P<0.001$, Figure 4).

\section{Clinical course of a study patient harboring a BRAF V600E mutation who received targeted therapy}

Recently, a patient diagnosed with lung adenocarcinoma without EGFR/ALK aberrations but harboring a BRAF V600E mutation received BRAF-mutation targeted therapy at our hospital. This case was not included in the main study cohort. The patient was a 47-year-old female, which had been diagnosed at our hospital with adenocarcinoma

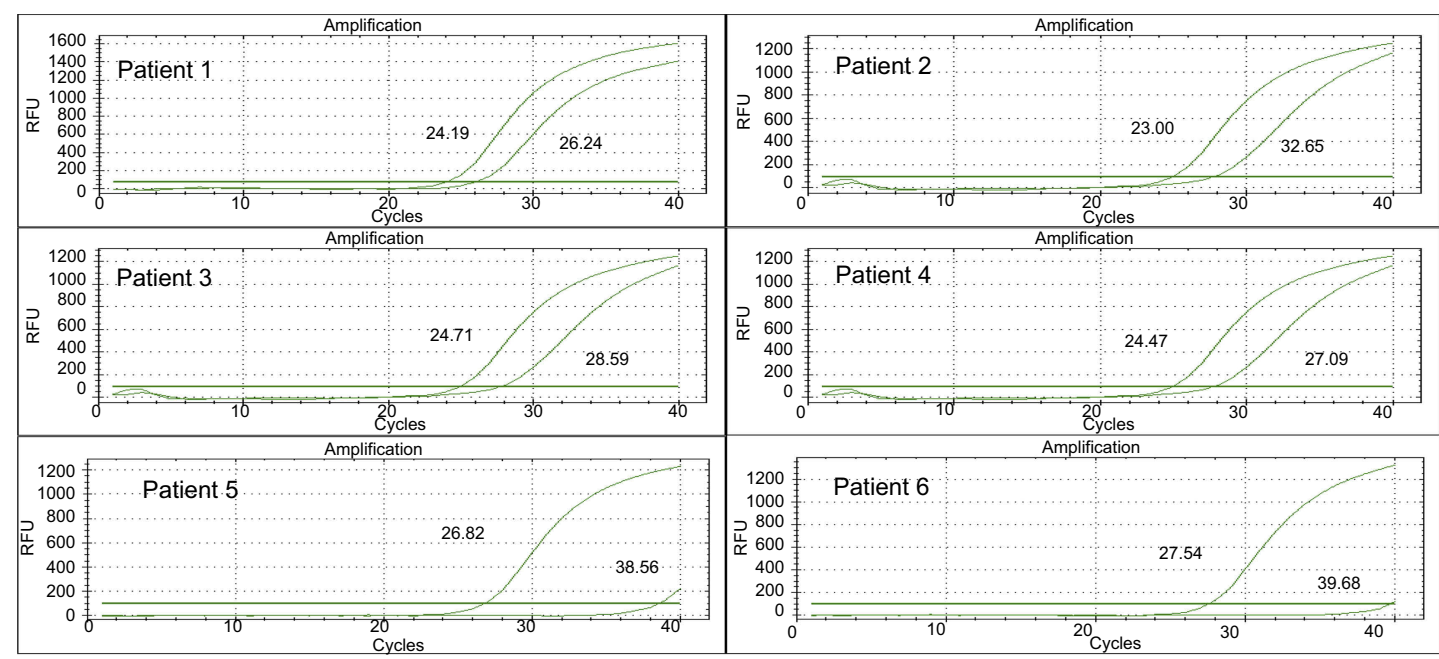

\begin{tabular}{|c|c|c|c|c|c|}
\hline \multirow{2}{*}{ Sample ID } & \multicolumn{2}{|c|}{$\mathrm{Ct}$} & \multirow{2}{*}{$\Delta \mathrm{Ct}-2^{\star \star}$} & \multirow{2}{*}{$\Delta \mathrm{Ct}-1^{*}$} & \multirow{2}{*}{ Result } \\
\hline & Non & BRAF & & & \\
\hline Patient 1 & 24.19 & 26.24 & 2.05 & 8.76 & V600E mutant \\
\hline Patient 2 & 23.00 & 32.65 & 9.65 & 2.35 & V600E mutant \\
\hline Patient 3 & 24.71 & 28.59 & 3.88 & 6.41 & V600E mutant \\
\hline Patient 4 & 24.47 & 27.09 & 2.62 & 7.91 & V600E mutant \\
\hline Patient 5 & 26.82 & 38.56 & 11.74 & -3.56 & Wild type \\
\hline Patient 6 & 27.54 & 39.68 & 12.14 & -4.68 & Wild type \\
\hline
\end{tabular}

Figure 3 Detection of the BRAF mutation in the study population. 
Table 2 Risk factors for mortality in the study patients assessed by using the Cox proportional hazards model

\begin{tabular}{|l|l|l|l|l|l|l|}
\hline \multirow{2}{*}{} & \multicolumn{2}{l|}{ Univariate analysis } & \multicolumn{2}{l|}{ Multivariate analysis } \\
\cline { 2 - 7 } & Hazard ratio & $\mathbf{9 5 \%} \mathbf{C l}$ & P-value & Hazard ratio & $\mathbf{9 5 \%} \mathbf{~ C l}$ & $\mathbf{P}$-value \\
\hline Age & 1.014 & $0.999-1.029$ & 0.067 & 1.000 & $0.985-1.017$ & 0.952 \\
Male sex & 1.503 & $1.083-2.087$ & 0.015 & 1.541 & $0.819-2.901$ & 0.180 \\
Ever-smoker & 1.343 & $0.956-1.885$ & 0.089 & 1.039 & $0.564-1.911$ & 0.903 \\
BRAF V600E mutation & 0.478 & $0.118-1.929$ & 0.300 & & $1.408-3.600$ & 0.001 \\
Best supportive care & 1.639 & $1.111-2.416$ & 0.013 & 2.252 & & \\
\hline
\end{tabular}

Table 3 Comparison of the baseline characteristics between the study population and patients with an EGFR mutation

\begin{tabular}{|c|c|c|c|c|}
\hline & Total & Study population & EGFR mutation population & $P$-value \\
\hline Patients number & 461 & 222 & 239 & \\
\hline Age, years & $60[52-69]$ & $63[57-7 \mid]$ & $57[50-64]$ & $<0.001$ \\
\hline Male sex & $24 I(52.3)$ & $153(68.9)$ & $88(36.5)$ & $<0.001$ \\
\hline Ever-smoker $(n=440)$ & $209(47.5)$ & $138(68.7)$ & 7I (29.7) & $<0.001$ \\
\hline Clinical stage & & & & $<0.001$ \\
\hline III & $82(17.8)$ & $54(24.3)$ & $28(11.7)$ & \\
\hline IV & $379(82.2)$ & $168(75.7)$ & $211(88.3)$ & \\
\hline
\end{tabular}

Note: Data are presented as a median (interquartile range), or number (\%), unless otherwise indicated.

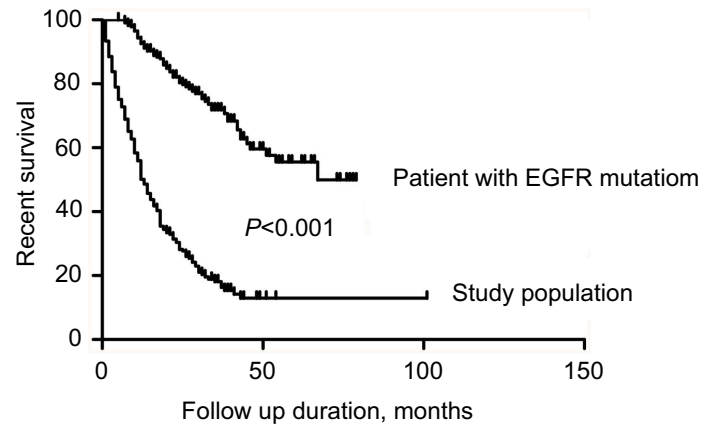

Figure 4 Kaplan-Meier survival curves comparing the study population with patients harboring an EGFR mutation.

in her left lower lobe five years prior and subsequently underwent a left lower lobe lobectomy. Her pathologic TNM stage was T2N0M0, with the cancer cells harboring a BRAF V600E mutation but not EGFR/ALK aberrations. Following the surgical intervention, she received adjuvant chemotherapy (cisplatin + vinorelbine). However, tumor recurrence occurred in both lung fields after approximately 3 years. She then received treatment with gemcitabine + cisplatin (5 months), pemetrexed (11 months), and nivolumab (9 months), but showed further disease progression. However, subsequent treatment with dabrafenib and trametinib after three previous lines of chemotherapy resulted in regression of the tumor after one month (Figure 5). At the time this paper was written, this patient had been

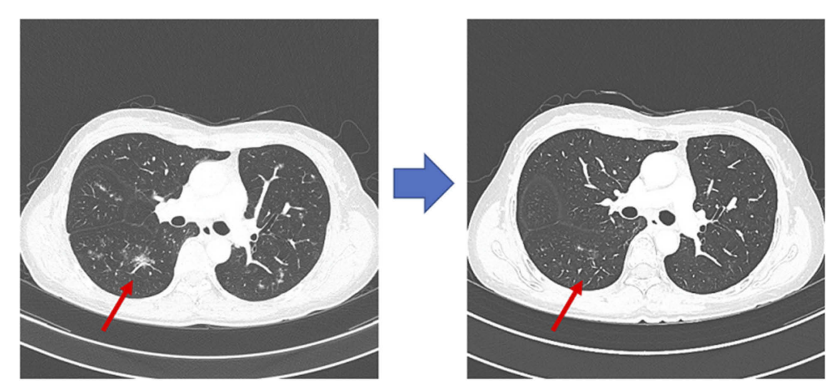

Figure 5 Response of a metastatic adenocarcinoma patient to dabrafenib and trametinib. The red arrows indicate the reduced mass.

undergoing treatment with dabrafenib and trametinib for eight months and being followed-up on.

\section{Discussion}

Our results showed that $4 / 222(1.8 \%)$ of the advanced lung adenocarcinoma patients without EGFR/ALK aberrations harbored a V600E BRAF mutation. Due to the rarity of this phenotype, the differences between the baseline characteristics and clinical outcomes of the patients included in the study with and without a BRAF mutation could not be readily evaluated. However, we further describe a case report of a patient with lung adenocarcinoma which had a BRAF mutation that was not included in the study cohort, who benefitted from BRAF-mutation targeted therapy. This is especially notable as adenocarcinoma patients 
without EGFR/ALK aberrations generally have limited treatment options.

The activation and disruption of specific signaling pathways are often associated with the development and progression of human cancer. ${ }^{18,19}$ The most well-known of these is the EGFR mutation and ALK rearrangement, which have led to the development of targeted therapies. ${ }^{5,20}$ In addition, there are various other molecular targets of interest for novel cancer therapy development such as ROS1, HER2, c-MET, RET, PIK3CA, FGFR1 and DDR2. ${ }^{21}$ BRAF mutations are among the relatively well known oncogenic drivers and directly interact with the MEK-ERK signaling cascade. ${ }^{22}$ Moreover, BRAF mutations have been presently reported in approximately 1$5 \%$ of NSCLCs (mainly in adenocarcinomas), ${ }^{11-15,23,24}$ which is consistent with the frequency found in our current study. To the best our knowledge, no prior study has investigated the prevalence of these variations in adenocarcinoma patients without EGFR/ALK aberrations.

All of the patients with a BRAF mutation in our current cohort harbored the V600E variant. In contrast to other malignancies, such as malignant melanoma, many previous studies have reported that only $50 \%$ of BRAF mutations in lung cancer are V600E. ${ }^{25-28}$ However, Villaruz et al reported that 17/21 lung cancer patients with a BRAF mutation harbored the V600E variant, which is concordant with our findings ${ }^{29}$ The discrepancies of these results may be due to differences in the study populations. Indeed, here, we only included patients with advanced adenocarcinoma and without EGFR/ALK aberrations, which may have affected our results. In addition, ethnic differences between studies may be a factor. Further studies of larger populations will be needed to confirm these findings.

The reported overall median survival rate of advanced or metastatic NSCLC patients with BRAF mutations has varied considerably in previous studies, ranging from 9 to 56 months. $^{27-31}$ This is likely due to differences between study populations, including the type of BRAF mutation, presence or absence of an EGFR mutation, and the targeted therapy administered. Although only 4 of the patients in our study cohort presented with a BRAF mutation, the median survival rate in these cases was 23 months, which is comparable to previous findings. The patients in this study received no targeted therapies, as they only became commercially available in 2017. Notably, one recent advanced lung cancer patient without EGFR/ALK aberrations, but with a BRAF mutation, participated in a clinical trial of the targeted therapy agents dabrafenib and trametinib at our hospital and showed a promising clinical outcome. There is now growing evidence that these drugs are effective in these types of cases. ${ }^{32,33}$ In a phase 2 study, 26 patients (33\%) treated with dabrafenib showed an investigator-assessed overall response among 84 patients with stage IV lung cancer and with a BRAF mutation. $^{32}$ Moreover, in another phase 2 study, 36 patients $(63.2 \%)$ treated with dabrafenib and trametinib showed an investigator-assessed overall response among 59 patients with stage IV lung cancer and with a BRAF mutation. Thus, these agents might become an important treatment option in the near future.

This study had some notable limitations. Firstly, due to the retrospective nature of our analysis, BRAF mutation analysis was not performed in all of the included advanced adenocarcinoma cases. This may have introduced a selection bias. Secondly, the patients included in the study patients were selected only from our own single tertiary referral center which may reduce the general applicability of our findings. Thirdly, as BRAF mutations were detected in only 4 of the patients included in the study $(1.8 \%$ of the total subjects), statistical comparisons could not be reliably performed. Finally, as our study only investigated BRAF mutations which showed low frequency in our study cohort, it is still necessary to investigate other gene mutations that might be clinically helpful. However, we believe that our current results enhance our understanding of the effect of BRAF mutations in advanced lung adenocarcinoma without EGFR/ALK aberrations, particularly in Asian populations.

\section{Conclusion}

In conclusion, BRAF mutations occur at a low frequency in advanced lung adenocarcinoma patients without EGFR/ ALK aberrations. However, BRAF-mutation targeted therapy might be effective in these unusual cases. As such, a larger scale study is needed in the near future to further evaluate this possibility.

\section{Abbreviation list}

EGFR, epidermal growth factor receptor; ALK, anaplastic lymphoma kinase; NSCLC, non-small cell lung cancer; BRAF, B-Raf and V-Raf murine sarcoma viral oncogene homolog; IQR, interquartile range; HR, hazard ratio.

\section{Ethics approval and informed consent}

The study protocol was approved by the Institutional Review Board of Asan Medical Center (approval number: 
2018-1109) and written informed consent for the use of tissue samples for clinical research was obtained from all patients or their appropriate family members. This study was conducted in accordance with the Declaration of Helsinki.

\section{Data availability}

The datasets used and analyzed during the current study are available from the corresponding author on reasonable request.

\section{Acknowledgments}

This was a collaborative study between Novartis Korea and Asan Medical Center. Novartis Korea contributed funding and participated in the manuscript writing. This study was supported by Novartis Korea.

\section{Author contributions}

The principle investigator (Chang-Min Choi) contributed to the entire study design. All authors contributed to data analysis, drafting or revising the article, gave final approval of the version to be published, and agree to be accountable for all aspects of the work.

\section{Disclosure}

Yeh Rim Kang is an employee of Novartis Korea. The authors report no other conflicts of interest in this work.

\section{References}

1. Siegel RL, Miller KD, Jemal A. Cancer statistics, 2019. CA Cancer J Clin. 2019;69(1):7-34. doi:10.3322/caac.21551

2. Mayekar MK, Bivona TG. Current landscape of targeted therapy in lung cancer. Clin Pharmacol Ther. 2017;102(5):757-764. doi:10.1002/cpt.810

3. Lynch TJ, Bell DW, Sordella R, et al. Activating mutations in the epidermal growth factor receptor underlying responsiveness of nonsmall-cell lung cancer to gefitinib. N Engl J Med. 2004;350(21):21292139. doi:10.1056/NEJMoa040938

4. Maemondo M, Inoue A, Kobayashi K, et al. Gefitinib or chemotherapy for non-small-cell lung cancer with mutated EGFR. $N$ Engl $J$ Med. 2010;362(25):2380-2388. doi:10.1056/NEJMoa0909530

5. Shaw AT, Kim DW, Mehra R, et al. Ceritinib in ALK-rearranged non-smallcell lung cancer. $N$ Engl J Med. 2014;370(13):1189-1197. doi:10.1056/ NEJMoa1311107

6. Pao W, Girard N. New driver mutations in non-small-cell lung cancer. Lancet Oncol. 2011;12(2):175-180. doi:10.1016/S1470-2045(10)70087-5

7. Leicht DT, Balan V, Kaplun A, et al. Raf kinases: function, regulation and role in human cancer. Biochim Biophys Acta. 2007;1773(8):11961212. doi:10.1016/j.bbamcr.2007.05.001

8. Davies H, Bignell GR, Cox C, et al. Mutations of the BRAF gene in human cancer. Nature. 2002;417(6892):949-954. doi:10.1038/nature00766

9. Flaherty KT, Puzanov I, Kim KB, et al. Inhibition of mutated, activated BRAF in metastatic melanoma. $N$ Engl $J$ Med. 2010;363 (9):809-819. doi:10.1056/NEJMoa1002011
10. Chapman $\mathrm{PB}$, Hauschild $\mathrm{A}$, Robert $\mathrm{C}$, et al. Improved survival with vemurafenib in melanoma with BRAF V600E mutation $N$ Engl J Med. 2011;364(26):2507-2516. doi:10.1056/ NEJMoa1103782

11. Ding L, Getz G, Wheeler DA, et al. Somatic mutations affect key pathways in lung adenocarcinoma. Nature. 2008;455(7216):10691075. doi: $10.1038 /$ nature 07423

12. Naoki K, Chen TH, Richards WG, Sugarbaker DJ, Meyerson M. Missense mutations of the BRAF gene in human lung adenocarcinoma. Cancer Res. 2002;62(23):7001-7003.

13. Li Z, Jiang L, Bai H, et al. Prevalence and clinical significance of BRAF V600E in Chinese patients with lung adenocarcinoma. Thorac Cancer. 2015;6(3):269-274. doi:10.1111/1759-7714.12177

14. Sasaki H, Kawano O, Endo K, et al. Uncommon V599E BRAF mutations in Japanese patients with lung cancer. J Surg Res. 2006;133(2):203-206. doi:10.1016/j.jss.2005.10.022

15. Li S, Li L, Zhu Y, et al. Coexistence of EGFR with KRAS, or BRAF, or PIK3CA somatic mutations in lung cancer: a comprehensive mutation profiling from 5125 Chinese cohorts. $\mathrm{Br} \mathrm{J}$ Cancer. 2014;110(11):2812-2820. doi:10.1038/bjc.2014.210

16. Koo HJ, Kim MY, Park S, et al. Non-Small Cell Lung Cancer with Resistance to EGFR-TKI Therapy: CT Characteristics of T790M Mutation-positive Cancer. Radiology. 2018;289(1):227-237.

17. Jeong D, Jeong $Y$, Lee $S$, et al. Detection of BRAF(V600E) mutations in papillary thyroid carcinomas by peptide nucleic acid clamp realtime PCR: a comparison with direct sequencing. Korean J Pathol. 2012;46(1):61-67. doi:10.4132/KoreanJPathol.2012.46.1.61

18. Nicholson KM, Anderson NG. The protein kinase B/Akt signalling pathway in human malignancy. Cell Signal. 2002;14(5):381-395.

19. Downward J. Targeting RAS signalling pathways in cancer therapy. Nat Rev Cancer. 2003;3(1):11-22. doi:10.1038/nrc969

20. Kobayashi S, Boggon TJ, Dayaram T, et al. EGFR mutation and resistance of non-small-cell lung cancer to gefitinib. $N$ Engl J Med. 2005;352(8):786-792. doi:10.1056/NEJMoa044238

21. Rothschild SI. Targeted therapies in non-small cell lung cancerbeyond EGFR and ALK. Cancers (Basel). 2015;7(2):930-949. doi:10.3390/cancers7020816

22. Wan PT, Garnett MJ, Roe SM, et al. Mechanism of activation of the RAF-ERK signaling pathway by oncogenic mutations of B-RAF. Cell. 2004;116(6):855-867.

23. Schmid K, Oehl N, Wrba F, Pirker R, Pirker C, Filipits M. EGFR/KRAS/ BRAF mutations in primary lung adenocarcinomas and corresponding locoregional lymph node metastases. Clin Cancer Res. 2009;15 (14):4554-4560. doi:10.1158/1078-0432.CCR-09-0089

24. Serizawa M, Koh Y, Kenmotsu H, et al. Assessment of mutational profile of Japanese lung adenocarcinoma patients by multitarget assays: a prospective, single-institute study. Cancer. 2014;120 (10):1471-1481. doi:10.1002/cncr.28604

25. Zheng G, Tseng LH, Chen G, et al. Clinical detection and categorization of uncommon and concomitant mutations involving BRAF. BMC Cancer. 2015;15:779. doi:10.1186/s12885-015-1584-3

26. Paik PK, Arcila ME, Fara M, et al. Clinical characteristics of patients with lung adenocarcinomas harboring BRAF mutations. $J$ Clin Oncol. 2011;29(15):2046-2051. doi:10.1200/JCO.2010.33.1280

27. Cardarella S, Ogino A, Nishino M, et al. Clinical, pathologic, and biologic features associated with BRAF mutations in non-small cell lung cancer. Clin Cancer Res. 2013;19(16):4532-4540. doi:10.1158/ 1078-0432.CCR-13-0657

28. Litvak AM, Paik PK, Woo KM, et al. Clinical characteristics and course of 63 patients with BRAF mutant lung cancers. $J$ Thorac Oncol. 2014;9(11):1669-1674. doi:10.1097/JTO.000000 0000000344

29. Villaruz LC, Socinski MA, Abberbock S, et al. Clinicopathologic features and outcomes of patients with lung adenocarcinomas harboring BRAF mutations in the Lung Cancer Mutation Consortium. Cancer. 2015;121(3):448-456. doi:10.1002/cncr.29042 
30. Brustugun OT, Khattak AM, Tromborg AK, et al. BRAF-mutations in non-small cell lung cancer. Lung Cancer. 2014;84(1):36-38. doi:10.1016/j.lungcan.2014.01.023

31. Tissot C, Couraud S, Tanguy R, Bringuier PP, Girard N, Souquet PJ. Clinical characteristics and outcome of patients with lung cancer harboring BRAF mutations. Lung Cancer. 2016;91:23-28. doi:10.1016/j.lungcan.2015.11.006

32. Planchard D, Kim TM, Mazieres J, et al. Dabrafenib in patients with BRAF(V600E)-positive advanced non-small-cell lung cancer: a single-arm, multicentre, open-label, phase 2 trial. Lancet Oncol. 2016;17 (5):642-650. doi:10.1016/S1470-2045(16)00077-2
33. Planchard D, Besse B, Groen HJM, et al. Dabrafenib plus trametinib in patients with previously treated BRAF(V600E)-mutant metastatic nonsmall cell lung cancer: an open-label, multicentre phase 2 trial. Lancet Oncol. 2016;17(7):984-993. doi:10.1016/S1470-2045(16)30146-2

\section{Publish your work in this journal}

OncoTargets and Therapy is an international, peer-reviewed, open access journal focusing on the pathological basis of all cancers, potential targets for therapy and treatment protocols employed to improve the management of cancer patients. The journal also focuses on the impact of management programs and new therapeutic agents and protocols on patient perspectives such as quality of life, adherence and satisfaction. The manuscript management system is completely online and includes a very quick and fair peer-review system, which is all easy to use. Visit http://www.dovepress.com/ testimonials.php to read real quotes from published authors.

Submit your manuscript here: https://www.dovepress.com/oncotargets-and-therapy-journal 